\section{UNDERESTIMATION OF SERUM IgG LEVELS IN PATIENTS WITH WALDENSTROM'S MACROGLOBULINAEMIA AND A HIGH IgM BURDEN}

Joanne McElroy $^{2}$, Fenfen Cai ${ }^{1,2}$, Suran L. Fernando ${ }^{1,2,3}$, Richard B. Fulton ${ }^{2}$

${ }^{1}$ Department of Clinical Immunology and Allergy, Royal North Shore Hospital, ${ }^{2}$ ImmunoRheumatology Laboratory, Pathology North, and ${ }^{3}$ Sydney Medical School - Northern, Sydney, NSW, Australia

Background: Immunoglobulins have been measured with the Abbott Architect turbidimetric assay since June 2014 at Pathology North, Sydney, replacing the Beckman Immage nephelometric method. A discrepancy in IgG levels was observed in a small subset of patients.

Aim: To determine the characteristics of a small set of test samples that demonstrated discrepant IgG results.

Method: Patient medical histories and immunoglobulin levels were reviewed. Samples were run on alternative nephelometric and turbidimetric IgG methods and compared with protein electrophoresis. 200 consecutive samples with IgG below the Architect reference range were run in parallel with the Immage.

Results: Correlation between the methods for low level IgG was r=0.97. From October 2014 to October 2015, 7170 immunoglobulin levels were analysed. Of these, 183 samples had IgM concentrations above $12.0 \mathrm{~g} / \mathrm{L}$ of which 36 (20\%) demonstrated underestimation of $\mathrm{IgG}$ by the Architect. All these samples were from 10 patients with Waldenstrom's macroglobulinaemia. The measured IgG levels were unreadable or extremely low $(<2.31 \mathrm{~g} / \mathrm{L})$.

Discussion: IgG levels in some patients with Waldenstrom's macroglobulinaemia and a high IgM level may be underestimated by the Architect. This may have clinical implications in diagnosing and managing coexisting immunoparesis.

\section{COMPARISON OF TWO ENA TESTING ALGORITHMS - ALBIA VS ELISA/LINE IMMUNOASSAY}

Dinusha Chandratilleke ${ }^{1,2}$, Roger Silvestrini ${ }^{1}$, Sue Culican ${ }^{1}$, David Campbell $^{1}$, Sanjay Swaminathan ${ }^{1,2,3}$, Ming-Wei Lin ${ }^{1,2}$

${ }^{1}$ Department of Immunopathology, ICPMR, Westmead Hospital, ${ }^{2}$ Faculty of Medicine, University of Sydney, and ${ }^{3}$ Faculty of Medicine, Western Sydney University, Sydney, NSW, Australia

Aims: Extractable nuclear antigen (ENA) antibody testing is often requested in patients with suspected connective tissue diseases. Most laboratories in Australia use a 2 step process involving a high sensitivity screening assay followed by a high specificity confirmation test. Multiplexing technology with Addressable Laser Bead Immunoassay (e.g., FIDIS) offers simultaneous detection of multiple antibody specificities (including dsDNA), allowing a single step screening and confirmation.

Methods: We compared our current diagnostic laboratory testing algorithm (Organtec ELISA screen, Euroimmun line immunoassay confirmation) and the FIDIS Connective Profile.

Results: 529 samples (443 consecutive +86 known autoantibody positivity) were run through both algorithms, and 479 samples $(90.5 \%)$ were concordant. 100 samples (18.9\%) detected the same autoantibody profile and 379 were concordant negative samples $(71.6 \%)$. The 50 discordant samples $(9.5 \%)$ were subdivided into 'likely FIDIS or current method correct' or 'unresolved' based on ancillary data. 25 samples remained 'unresolved' and were sub-classified into 'potentially' vs 'potentially not' clinically significant based on the change to clinical interpretation. Only nine samples $(1.7 \%)$ were deemed to be 'potentially clinically significant'.

Discussion: Overall, we found that the FIDIS Connective Profile ENA kit is non-inferior to the current ELISA screen/LIA characterisation. Reagent and capital costs may be limiting factors in using the FIDIS, but potential benefits include a single step analysis and simultaneous detection of dsDNA antibodies.

\section{CHARACTERISATION OF PERIPERHAL B CELL PHENOTYPES IN PATIENTS WITH SARCOIDOSIS}

Jocelyn X. Jiang ${ }^{1,2}$, Carolyn Hawkins ${ }^{1,2}$, Jennifer Stapleton ${ }^{1}$, Matthew Cook ${ }^{1,2}$

${ }^{1}$ ACT Pathology, and ${ }^{2}$ Department of Immunology, The Canberra Hospital, Garran, ACT, Australia

Background: Sarcoidosis is a chronic inflammatory disorder of unknown cause characterised by poorly defined immune deficiency.

Aim: Characterise circulating B cell phenotypes in patients diagnosed with sarcoidosis.

Methods: We undertook an audit of B cell phenotyping by flow cytometry in sarcoidosis patients performed between 2008-2015 in our diagnostic laboratory. We analysed total B cells, memory B cells (CD19+CD27+) and class-switched memory B cells (CD19+CD27+IgM-IgD-) to yield a Freiburg classification. These data were analysed according to patient immunoglobulin, ESR, CRP and ACE levels.

Results: We identified 34 patients with a diagnosis of sarcoidosis who had undergone B cell phenotyping. $44 \%$ of patients had a deficiency of class-switched memory B cells $(<0.4 \%)$ and were classified as Freiburg class I with $32 \%$ class Ib and $12 \%$ class Ia. Proportions of class-switched memory B cells $<0.4 \%$ were associated with higher IgG levels.

Discussion: We identified a high incidence of B cell subset deficiencies in patients with sarcoidosis. Both sarcoidosis and common variable immunodeficiency are associated with granulomas, but in sarcoidosis, we observed a correlation between deficiency of switched memory B cells with increased serum IgG. This suggests that changes in B cells might reflect disease activity.

\section{DESCRIPTIVE COHORT OF ANA NEGATIVE, ENA POSITIVE PATIENTS OVER A 7 YEAR PERIOD}

Benjamin McGettigan, Christine Bundell,

Andrew McLean-Tooke

PathWest QEII, Perth, WA, Australia

Aim: To identify and describe patients with negative ANA and positive ENA antibodies.

Methods: Information on patients who had a negative ANA and positive ENA between 1st January 2007 and 1st Jan 2014 was 
extracted from the PathWest laboratory information system and clinical database. ANA assays were performed by indirect immunofluorescence. ENA requests were screened by ELISA and characterised by immunoblot.

Results: We identified 643 patients with an average age of 54, of whom 174 were male (27\%). There were 1111 antibodies found in total. Ro-52 antibodies were the most common (29\%), followed by SSA detected by IIF (19\%), Ro-60 (18\%), SSB $(8 \%)$, Histone (4\%), Scl-70 (4\%), Smith (4\%) and Jo-1 (4\%). Other antibodies were all $<1 \%$ (CenpB, RNP and Ribo-P). In total there were 459 patients that had only 1 ENA specificity (71\%), 121 had 2 (19\%), 55 had $3(9.2 \%)$ and 8 had 4 (1.2\%). Multiple clinic-pathological associations were found which will be discussed.

Discussion: Previous studies have identified patients with positive ENA and clinical autoimmune disease despite negative ANA. ${ }^{1}$ Our study confirms this finding within our referral population. Patients with a high pre-test probability for autoimmune disease should have ENA testing performed regardless of ANA results.

Reference

1. Davis JM 3rd, Moder KG, Homburger HA, et al. Clinical features of 39 patients with antibodies to ENA despite negative ANA: evidence for autoimmunity, including neurologic and connective tissue diseases. Medicine 2005; 84: 208-17.

\section{WITH 'COMPLEMENTS' TO OCCAM'S RAZOR}

Grace Thompson ${ }^{1}$, Mina John ${ }^{2,3}$

${ }^{1}$ Sir Charles Gairdner Hospital, Department of Immunology, ${ }^{2}$ Royal Perth Hospital Department of Immunology, and ${ }^{3}$ The Institute of Immunology and Infectious Diseases, Murdoch University, WA, Australia

We describe a case of a previously well 43-year-old woman with pre-existing mild Sjogrens syndrome who presented with a severe, rapidly progressive SRP positive necrotising autoimmune myositis (NAM) causing eventual quadriparesis and treated with multiple immunosuppressives and Rituximab. In the course of this illness she developed a fulminant non-shiga toxin associated haemolytic uraemic syndrome (HUS) that was refractory to plasmapharesis and was only arrested with the C5a inhibitor eculizumab. Concurrent atypical HUS and NAM has not been described previously, both being rare disorders but possibly unified by involvement of the alternate complement pathway. In aHUS, uncontrolled complement activation is usually secondary to underlying genetic defects in regulatory proteins (Factor $\mathrm{H}$, Factor $\mathrm{I}$ and membrane cofactor protein) or defects in Factor B, C3 with a 'second hit' caused by autoantibodies against complement regulatory proteins or infectious triggers. Investigations to date in this patient have shown low Factor I levels but known Factor I mutations have not been detected to date. We explore the possible ways in which autoimmunity or SRP-associated complement activation may have played a role in susceptibility to aHUS in this case and broader implications for complement-mediated disease.

\section{VALIDATION OF A HLA-B*15:02 SCREENING METHOD IN THE PREVENTION OF CARBAMAZEPINE - INDUCED SEVERE CUTANEOUS ADVERSE REACTIONS}

Christopher Vidal $^{1}$, Dinh Van Nguyen ${ }^{1,2,3}$, Jamma Li $^{1,4}$, Fen Cai ${ }^{1,4}$, Richard B. Fulton ${ }^{1}$, Hieu Chi Chu ${ }^{5}$, Anne Proos ${ }^{6}$, Suran L. Fernando ${ }^{1,2,4}$

${ }^{1}$ ImmunoRheumatology Laboratory, Pathology North, ${ }^{2}$ Sydney Medical School - Northern, University of Sydney, Sydney, NSW, Australia; ${ }^{3}$ Department of Allergy and Clinical Immunology, Hanoi Medical University, Hanoi, Vietnam; ${ }^{4}$ Department of Clinical Immunology and Allergy, Royal North Shore Hospital, Sydney, NSW, Australia; ${ }^{5}$ Center of Allergology and Clinical Immunology, Bach Mai Hospital, Hanoi, Vietnam; and ${ }^{6}$ Department of Molecular Genetics, Pathology North, Sydney, NSW, Australia

Background: HLA-B*15:02 is strongly associated with carbamazepine (CBZ)-induced Stevens-Johnson syndrome/toxic epidermal necrolysis in Asians, thus screening for this allele is recommended. Our aim was to develop a widely applicable and cost-effective in-house assay for the detection of HLA-B*15:02. Methods: 103 DNA samples were tested for validation. A novel multiplex SYBR real-time PCR using allele specific primers for HLA-B*15:02 was developed. Actin was used as an internal control. The dissociation curve was used to determine HLAB*15:02 and actin. Results were compared with those obtained by Luminex SSO/SBT/SSP.

Results: From 103 samples tested, HLA-B*15:02 allele was detected in 10 samples showing two peaks at $91.3^{\circ} \mathrm{C}\left(\mathrm{B}^{*} 15: 02\right)$ and $80.9^{\circ} \mathrm{C}$ (actin), respectively. One false positive sample was detected when compared to Luminex. Our assay has $100 \%$ sensitivity, 99\% specificity, PPV: $90 \%$ and NPV: 100\%, respectively. The lowest limit of detection is $0.5 \mathrm{ng} / \mu \mathrm{L}$ DNA, rendering it suitable for low yield-samples. The unit cost is half compared to that of a commercial assay.

Conclusion: We have developed a rapid, robust and costeffective method that produces no false negative results that can be used for the screening of HLA-B*15:02 prior to the initiation of treatment with CBZ in individuals with Asian ancestry.

\section{A VERY RARE CASE OF INFECTIVE ENDOCARDITIS}

Eric Chan $^{1}$, Chris Hopkins ${ }^{2}$

${ }^{1}$ Department of Microbiology, LabPlus, Auckland City Hospital, and ${ }^{2}$ Infectious Disease Department, Northshore Hospital, Auckland, New Zealand

Introduction: Microascus cinereus fungal endocarditis in an immunocompetent person is a very rare occurrence. This dematiaceous fungus is normally found in soil and stored grains. Case presentation: A normally well 64-year-old Indian male had for 5 months vague symptoms of breathlessness with on/off mild fever. Previous illnesses included diabetes mellitus, pulmonary nodules, cardiomyopathy, and heart block with permanent pace-maker insertion in India. He presented after 5 months 